\title{
The Use of Egocentric and Allocentric Reference Frames in Static and Dynamic Conditions in Humans
}

\author{
Sofiia MORARESKU ${ }^{1,2}$, Kamil VLCEK ${ }^{1}$ \\ ${ }^{1}$ Laboratory of Neurophysiology of Memory, Institute of Physiology of the Czech Academy of \\ Sciences, Prague, Czech Republic, ${ }^{2}$ Third Faculty of Medicine, Charles University, Prague, Czech \\ Republic
}

Received June 12, 2020

Accepted July 28, 2020

Epub Ahead of Print September 9, 2020

\section{Summary}

The dissociation between egocentric and allocentric reference frames is well established. Spatial coding relative to oneself has been associated with a brain network distinct from spatial coding using a cognitive map independently of the actual position. These differences were, however, revealed by a variety of tasks from both static conditions, using a series of images, and dynamic conditions, using movements through space. We aimed to clarify how these paradigms correspond to each other concerning the neural correlates of the use of egocentric and allocentric reference frames. We review here studies of allocentric and egocentric judgments used in static two- and three-dimensional tasks and compare their results with the findings from spatial navigation studies. We argue that neural correlates of allocentric coding in static conditions but using complex three-dimensional scenes and involving spatial memory of participants resemble those in spatial navigation studies, while allocentric representations in two-dimensional tasks are connected with other perceptual and attentional processes. In contrast, the brain networks associated with the egocentric reference frame in static two-dimensional and three-dimensional tasks and spatial navigation tasks are, with some limitations, more similar. Our review demonstrates the heterogeneity of experimental designs focused on spatial reference frames. At the same time, it indicates similarities in brain activation during reference frame use despite this heterogeneity.

\section{Key words}

Spatial Processing • Functional Neuroimaging • Reference frames - Allocentric • Egocentric

\section{Corresponding authors}

S. Moraresku and K. Vlcek, Laboratory of Neurophysiology of Memory, Institute of Physiology of the Czech Academy of Sciences, Videnska 1083, 14220 Prague, Czech Republic. E-mail: sofiia.moraresku@fgu.cas.cz and kamil.vlcek@fgu.cas.cz

\section{Introduction}

The concept of spatial reference frames is important for studying spatial cognition in humans as it is involved in spatial memory, perception, performing actions in space, and navigation. Two main classes of reference frames have been described: egocentric and allocentric. Various experimental paradigms have been used in neuroimaging and electrophysiological studies to investigate neural correlates of the use of spatial reference frames. Part of them explored the nature of allocentric and egocentric coding using spatial tasks such as direction or distance judgments in static images, either simple two-dimensional ones containing a few objects or more complex three-dimensional scenes from virtual reality environments. Another broad spectrum of studies aimed to investigate how humans use and manipulate spatial reference frames in dynamic conditions during navigation. These studies employed virtual environments such as analogies of the Morris water maze, virtual cities or tunnels, or mental navigation. Additional ones studied the effect of brain lesions in real space tasks. It is not obvious how these different approaches correspond to each other in terms of neural correlates. Here, we try to 
fill the gap in understanding of this issue. In the following text, we provide an overview of brain activity studies of egocentric and allocentric coding in static twodimensional tasks, then move on to studies using complex three-dimensional scenes. Finally, we compare their results with the findings from spatial navigation studies.

The search for relevant papers was performed on the PubMed database. As our main aim was the comparison of neural correlates of allocentric and egocentric reference frames use in static and dynamic conditions in humans, we included only studies analyzing brain activation using various neuroimaging methods such as fMRI, PET or EEG in humans. We excluded animal studies and human studies reporting only behavioral results. Except for brain lesion studies mentioned in the review, our search focused mainly on studies with the participation of healthy and young adults.

\section{Types of spatial reference frames}

According to Klatzky (1998), a reference frame is defined as a system of reference to represent the object's and subject's spatial positions. Parameters defining a reference frame include its origin, orientation, and scale. Two basic types have been suggested, based on where the origin is anchored (O'Keefe and Nadel 1978). In an egocentric reference frame, the locations of objects are coded in relation to the observer. On the contrary, allocentric coding is independent of the subject's position and based on inter-objects relations and relations between objects and global landmarks (O'Keefe and Nadel 1978, Klatzky 1998).

Each spatial reference frame could be divided into various subtypes. According to recent fMRI studies, several areas in the parietal cortex are associated with different egocentric reference frames. The eye-centered coordinates can be used for visual perception or spatial attention, as shown in the study by Medendorp et al. (2003). They identified a bilateral region located at a medial branch of the intraparietal sulcus (IPS), which monitors and updates spatial goals in the gaze-centered reference frame. In a delayed-saccade task, participants had to make a saccade towards the remembered location of the target, which was presented before left or right from the fixating point. The authors found topographical activation in the area near IPS, which responded more on the left side for saccades towards rightward goal locations and more on the right side for saccades towards leftward goal locations. This activation was in the gaze-centered reference frame as the body and head reference frames were fixed. The hand and body-centered reference frames are used for reaching near targets (Makin et al. 2007, Beurze et al. 2010), while the face-centered representation in the parietal face area allows coordinated head movements like eating or avoiding head collisions (Sereno and Huang 2006). However, in most studies focused on egocentric and allocentric representations, authors do not distinguish between these egocentric subtypes and mostly eye- or head-centered can be implied from the experimental design.

Regarding allocentric reference frame, in a general sense, it is defined as coding the location of an object relative to other objects or landmarks in the environment, independently from the viewer's position (O'Keefe and Nadel 1978, Klatzky 1998). Thus, this definition combines coding of local, object-to-object spatial relationships, which can be used in more general attentional and visuomotor control processes, with the coding of global spatial relationships between objects and landmarks in the environment used mainly for navigation. The first type of coding has been called 'object-centered'; it is essential for orienting in near space and representing in memory the relative location of visible and invisible objects (Easton and Sholl 1995). Our knowledge about its brain representation is closely associated with studies of brain-damaged patients suffering from object-centered neglect, expressed in the ignoring of the half of objects, contralateral to the lesion site, independently from the patient's midline (Chechlacz et al. 2012, Kenzie et al. 2015, Demeyere and Gillebert 2019). Apart from its engagement in spatial attention, it was also shown that the 'object-centered' reference frame could serve for visuomotor control, reaching and grasping (Honda et al. 1998, Patchay et al. 2006, Chen et al. 2014). For example, it could be involved in the initial localization of the part of an object relative to another object in order to grab it properly, which occurs already in egocentric coordinates. As for the second type of coding, it has been called 'world-centered' or 'environment-centered' (Committeri et al. 2004, Rosenbaum et al. 2004, Sulpizio et al. 2013, Wolbers and Wiener 2014, Ekstrom et al. 2017) as spatial locations of objects are coded with respect to some fixed characteristics of the environment. We use this global spatial coding mostly for the planning of a novel trajectory in the environment. 


\section{Two-dimensional studies}

The first line of research (Galati et al. 2000, Neggers et al. 2006, Chen et al. 2012, Saj et al. 2014, Liu et al. 2017, Ruotolo et al. 2019) employed perceptual spatial judgments in two-dimensional images. The experimental design included simple two-dimensional tasks, as a rule, consisting of basic objects such as bars, points, or circles. In an allocentric condition, participants judged distance or direction relative to the middle of an object or stimulus, while an egocentric condition was expressed in making distance or direction judgments relative to the midsagittal plane of participant's body. The experimental design generally did not allow distinguishing between eye-, head- and body-centered reference frames as the participant's body and head were in a fixed position during the whole experiment, and participants had to fixate the center of the screen before each trial. These tasks are similar conceptually to the line bisection test, which is commonly used to assess spatial impairments in patients suffering from unilateral or hemispatial neglect (Schenkenberg et al. 1980, Ishiai et al. 1989).

Galati and his colleagues (2000), for instance, used visuospatial tasks with left-right judgments about vertical bars intersecting a horizontal line. In the egocentric condition, participants had to determine whether the vertical line was on the right or the left from their body midsagittal plane. In the allocentric condition, participants had to determine whether the vertical line was on the right or the left from the middle of the horizontal line. In control non-spatial condition, participants had to judge the color of the vertical bar. Other studies followed a similar paradigm with various two-dimensional (Neggers et al. 2006, Ruotolo et al. 2019) or even three-dimensional objects (Chen et al. 2012, Liu et al. 2017) but still without any background making judgments about the position of the fork with respect either to the center of the plate or to the midsagittal of participant's body.

The results of these fMRI studies demonstrate a common pattern of activation in allocentric and egocentric spatial tasks compared with non-spatial. In particular, either the areas in the frontal cortex, such as the middle or superior frontal gyrus (Neggers et al. 2006), or in the parietal cortex, especially the superior or inferior parietal lobule (Chen et al. 2012, Saj et al. 2014), or both (Galati et al. 2000, Liu et al. 2017) were active during the spatial conditions. Direct comparisons between spatial conditions revealed, in general, a wider activation during the egocentric task than in allocentric. Moreover, egocentric judgments elicited response selectively in parts of the fronto-parietal network such as the superior parietal lobule, precuneus, superior, middle and inferior frontal gyri predominantly in the right hemisphere (Galati et al. 2000, Chen et al. 2012, Saj et al. 2014, Liu et al. 2017, Ruotolo et al. 2019). This activity associated with the egocentric reference frame is in line with findings from studies of patients suffering from unilateral spatial neglect. The most severe impairments were caused by lesions in the right hemisphere (Vallar 2007, Grimsen et al. 2008). In particular, lesions to the areas of the dorsal stream such as the right superior and middle frontal gyri, as well as to the perisylvian parts of the pre-, postcentral and supramarginal gyri resulted in egocentric impairments which were expressed in patient's inability to perceive space contralateral to their lesion (Grimsen et al. 2008, Chechlacz et al. 2012).

The reverse contrast, allocentric versus egocentric condition, revealed a common pattern of activation only in some studies. Although there was the trend for activation in the allocentric compared to the egocentric condition in the lingual gyrus and region around the tail of the right hippocampus (Galati et al. 2000) or in the middle frontal gyrus (Neggers et al. 2006), significant activation was observed only in the left inferior temporal gyrus (Saj et al. 2014) and bilaterally in the cuneus, calcarine sulcus and lingual gyrus (Ruotolo et al. 2019). In other cases, there was no significant activation at all (Chen et al. 2012, Liu et al. 2017). Despite the absence of activation in the allocentric versus egocentric contrast in some studies, other studies suggest a general pattern of activation for the processing of allocentric information in the ventral occipitotemporal cortex.

The difference in the significance of activation for the allocentric condition in the ventral areas could be explained by different applied experimental paradigms. For instance, Saj et al. (2014) used a pair of various stimuli (a dot and a vertical bar) and asked the subjects not to make left/right judgments (in contrast to Galati et al. 2000) but rather to establish whether the vertical bar was aligned with the dot (allocentric task) or whether it was aligned with the body midline longitudinal axis (egocentric task). In this case, tasks could be more distinguishable as participants estimated exact spatial relations rather than made relative distance judgments. As a result, Saj et al. (2014) observed significant activation 
in the ventral area, in particular, in the inferior temporal gyrus in the allocentric task. Galati et al. (2000) also found activation in the ventral occipitotemporal cortex, specifically in the lingual gyrus and hippocampus, but it did not reach statistical significance. This observed pattern of activation is partly in agreement with findings from studies of patients suffering from object-centered allocentric neglect. Patients who ignore the half of objects, contralateral to the lesion site, independently of their position, often have lesions in the ventral occipitotemporal areas (Grimsen et al. 2008, Chechlacz et al. 2012).

Other studies explored neural correlates of visuomotor tasks performed either in egocentric or allocentric reference frames in response to visual stimuli presented in two-dimensional images (Honda et al. 1998, Chen et al. 2014, Chen et al. 2018). The first study (Honda et al. 1998) used the following experimental paradigm: first, two polygons with a target spot inside each of them were presented in two of four corners of the screen (the location of objects and target spots were different each trial); after a delay, a cue in the form of one of two polygons without the target spot appeared in the center of the screen; in response to this cue, participants were required to move the joystick either in the remembered direction of the relative location of the target spot within the object (allocentric condition) or in the remembered direction of the object's location relative to the center of the screen (egocentric condition). Using PET, authors observed common activation in the posterior parietal cortex, anterior supplementary motor areas, ventral premotor cortex, and dorsolateral prefrontal cortex for allocentric and egocentric conditions compared with control (fixed response in constant direction). In the allocentric condition, activation in the ventral occipitotemporal region, namely in the inferior occipital and fusiform gyri, was additionally found, which is partly in agreement with findings from studies using perceptual spatial judgments (Saj et al. 2014, Ruotolo et al. 2019).

A similar, more recent study (Chen et al. 2014) examined reaching the locations of remembered targets in allocentric and egocentric reference frames. First, they presented two visual stimuli the same for all conditions: one as a landmark and another as a target for upcoming reaching. Then a delay phase was introduced when participants had to remember the location of a reach target. After that, only the landmark was presented either at the same or at a different position and then disappeared. Finally, participants had to reach the location of the remembered target relative to the gaze, which was fixed (egocentric condition) or relative to the landmark (allocentric condition). Audio instruction about which reference frame to use was given at the beginning of each trial. During the delay phase, the authors observed higher activation in the parieto-frontal network, including the dorsal premotor cortex, mid-posterior IPS and superior parieto-occipital cortex, for the egocentric task and higher activation in areas of the occipital and temporal lobes for the allocentric task, namely in the lingual gyrus, cuneus and inferior temporal gyrus. Authors suggested that allocentric representations of the target in the inferior temporal gyrus are somehow further transformed into the planning of reaching in the egocentric coordinates in the parieto-frontal network. To confirm this suggestion, Chen et al. (2018) designed another experiment in which participants always had to remember the position of a target in allocentric coordinates with the following egocentric reaching. They identified four areas in the parietal and frontal cortex, such as the right precuneus, right pre-supplementary motor area, and bilateral dorsal premotor cortex, which could participate in the conversion of allocentric representations of the remembered target to the egocentric reaching.

In summary, egocentric representations used in perceptual spatial judgments are similar to those involved in remembering the target for reaching and recruit the parieto-frontal network. Allocentric representations recruit specific areas of the ventral occipitotemporal cortex, including the inferior temporal, inferior occipital, lingual and fusiform gyri and cuneus (Honda et al. 1998, Chen et al. 2014, Saj et al. 2014, Ruotolo et al. 2019), also common for different types of tasks, although they were not active in all cases. The fact that visual processing areas of the occipital and temporal lobe are involved in allocentric representations is congruent with a function of the ventral visual stream in the perception of objects, the extraction of their characteristics and relations, in the model suggested by Goodale and Milner (1992).

\section{Three-dimensional studies}

Another line of research (Committeri et al. 2004, Bastin et al. 2013, Sulpizio et al. 2013) explored neural correlates of egocentric and allocentric coding using complex three-dimensional scenes reconstructed from the real-world environment. In comparison to previously 
mentioned studies, this approach allows investigating the use of spatial reference frames in conditions close to real navigation but still without movements in space. The experimental design included both allocentric condition types: object-centered and world-centered. In the former condition, participants encoded the location of the target object relative to other unstable objects ignoring the surrounding environment. The latter condition, by contrast, required participants to encode the position of the target object relative to the stable characteristics of the scene. The egocentric condition depended on the current position of the participants, i.e. the current point of view in the scene, and thus, differed from that in twodimensional studies.

In two studies (Committeri et al. 2004, Bastin et al. 2013), the authors used a realistic virtual reconstruction of an area in front of a three-winged palace with a fountain. This area also contained several objects such as garbage cans and a red ball, which changed their locations each trial. In the egocentric condition, participants should indicate which of two garbage cans was closer to their current position. In the object-centered allocentric condition, participants should report which of two garbage cans was closer to the red ball. It is worth noting that in previously mentioned studies, the objectcentered condition was limited mostly by left-right distance judgments and, thus, presented only one dimension of space, whereas here objects were put in a three-dimensional scene and distance judgments included another aspect of space such as depth. In the world-centered condition, participants should report which of two garbage cans was closer to the central wing of the palace, which was not visible from their current viewpoint. Accordingly, the second condition required participants to make a mental reconstruction of the entire environment to locate the central wing of the palace relative to some fixed features of the environment, including lateral wings of the palace. The control nonspatial task required participants to determine which of two garbage cans was lying on the ground. In the third study (Sulpizio et al. 2013), the task for participants was to recognize a change in the target object position between two presented scenes, relative to their view, relative to the furniture on the central rotatable carpet arena or relative to the whole room.

Comparing spatial conditions with non-spatial, authors found common activation in the frontal, parietal, and occipital lobes (Committeri et al. 2004, Sulpizio et al. 2013). Besides, the overall activation was higher for both allocentric conditions (object- and world-centered), in contrast to studies using simple two-dimensional tasks, where the trend was the opposite: allocentric tasks even did not show significant activation in some cases. Furthermore, each spatial reference frame showed a different pattern of activation. The fronto-parietal regions, including the precuneus, supramarginal gyrus, posterior intraparietal sulcus and temporo-parieto-occipital junction, were involved in the egocentric frame transformations in one of the studies (Sulpizio et al. 2013), while only the right superior and inferior frontal gyri were more involved in the egocentric condition in Committeri et al. (2004) and Bastin et al. (2013) studies. Surprisingly, in these two studies, the authors did not observe higher activation in parietal regions for egocentric than for other spatial conditions. The egocentric distance judgment was made in relation to the participant's position as a whole rather than to a particular body part, which could result in the reduction of parietal involvement as authors suggested (Committeri et al. 2004).

The object-centered condition was associated with increased activity in the bilateral lateral occipitotemporal cortex, including inferior temporal and occipital gyri (Committeri et al. 2004) and temporoparieto-occipital junction (Sulpizio et al. 2013). The activation pattern in the object-centered allocentric condition overlapped with allocentric conditions in twodimensional tasks, although not completely. The common lateral occipitotemporal activity, including inferior temporal and occipital gyri, together with the observation of patients suffering from object-centered neglect after similarly located brain lesions, suggests the similarity of object-based space coding in two and three-dimensional static scene tasks.

Finally, the world-centered condition was associated with the highest activity in the retrosplenial cortex (RSC) and in the ventromedial occipitotemporal cortex (Committeri et al. 2004, Bastin et al. 2013, Sulpizio et al. 2013), which included the medial fusiform gyrus, the lingual gyrus, and the posterior part of the parahippocampal gyrus. The part of activation in the ventromedial occipitotemporal cortex overlapped with activation observed during the allocentric task in twodimensional studies, namely in the lingual and fusiform gyri (Honda et al. 1998, Chen et al. 2014, Ruotolo et al. 2019). Activation in the parahippocampal gyrus overlapped with the localization of the parahippocampal place area (PPA, Epstein and Kanwisher 1998) that 
responds preferentially to images of buildings and threedimensional scenes. Its activation is possibly related to the required focus on the whole environmental geometry during the world-centered condition. Late PPA response during the world-centered condition was also documented in a follow-up intracranial study (Bastin et al. 2013).

At the same time, the RSC activity relates probably to the required reorientation (Julian et al. 2018), as the changed viewpoint during each trial was crucial for world-centered responses but not for other conditions. Besides, the precuneus was active only in the worldcentered condition (Committeri et al. 2004), which required participants to mentally imagine the whole environment in order to localize the central wing of the palace. Its involvement could be explained by the process of mental imaging as shown in other studies (Fletcher et al. 1996, Ghaem et al. 1997, Malouin et al. 2003), in particular, it was active during motor imagery (Malouin et al. 2003) and mental navigation (Ghaem et al. 1997). On the other hand, precuneus activation could be associated with the translation between egocentric and allocentric representations: in the world-centered condition, participants needed to estimate their position and heading relative to the invisible central wing of the palace. The involvement of the precuneus in the egocentric-allocentric transformation was shown in other spatial tasks (Dhindsa et al. 2014) as well as in the reverse translation of allocentric representations of the remembered target to the egocentric reaching (Chen et al. 2018).

Of note is the lack of specific hippocampal activity in the world-centered condition. This condition partially resembles the allocentric navigation in Morris water maze in its design where the hippocampal activation has been documented (Parslow et al. 2004, Rodriguez 2010). However, the hippocampus is probably more essential for trajectory planning (Spiers and Maguire 2007) and more complex topographical tasks involving larger environments (Maguire et al. 1998) than simple distance estimation. Also, the hippocampus might rather be involved in integrating information about multiple spatial locations (Zhang and Ekstrom 2013), which was not required during the world-centered allocentric task.

\section{Spatial navigation studies}

We reviewed several studies investigating neural correlates of allocentric and egocentric representations in static conditions; however, in everyday life, humans manipulate these reference frames mostly while navigating and orienting in space. So, in the following section, we discuss the neural activation during the use of allocentric and egocentric reference frames in dynamic conditions, during navigation, and compare it with that in static conditions.

In spatial navigation studies, egocentric and allocentric strategies have been defined by the prevailing use of the corresponding reference frame. The egocentric strategy is based on the assessment of distances and directions from the body axes to the current goal and local landmarks. By contrast, people adopting an allocentric strategy establish spatial relationships between landmarks in the environment and their navigational goal independently from their position.

One of the popular spatial navigation tasks used in humans is the analog of the Morris water maze (MWM) originated from rodent studies. In the virtual analog of the MWM, in the allocentric condition, participants should find the invisible goal in the virtual circular arena using landmarks on the walls (Parslow et al. 2004, Rodriguez 2010) or object cues in its overhead version (Feigenbaum and Morris 2004) while their starting point is changing across trials. In the egocentric condition, the relationship between the starting position and invisible goal location is the same across trials, but landmarks on the walls (Parslow et al. 2004) or locations of object cues (Feigenbaum and Morris 2004) are changing. So, participants should ignore them and search for the invisible goal with respect to their starting position. Also, real-space models of the MWM were developed to assess allocentric memory in neurology patients (Bohbot et al. 1998, Kalova et al. 2005, Bohbot and Corkin 2007, Amlerova et al. 2013). In these models, subjects search for the hidden goal while orienting themselves relative to the room landmarks or start position in the allocentric and egocentric condition, respectively, similarly to the virtual analog models.

Another often used experimental paradigm is navigation in the large-scale environment as the virtual maze or city (Aguirre et al. 1996, Maguire et al. 1998, Hartley et al. 2003, Jordan et al. 2004, Iaria et al. 2007, Spiers and Maguire 2007, Weniger et al. 2009, Weniger et al. 2010). A typical example of the allocentric task is to find the shortest way between two locations, often between the starting and final position in the virtual maze (Jordan et al. 2004) or between two landmarks located far from each other in the virtual city (Maguire et al. 1998, 
Spiers and Maguire 2007, Iaria et al. 2007). Also, using detours has been employed as an example of allocentricbased navigation if the original way was blocked (Maguire et al. 1998, Spiers and Maguire 2007). In both cases, participants need to use a cognitive map of the environment to resolve a task successfully. In contrast, in egocentric tasks, participants are often required to navigate along habitual routes or follow trails or arrows along the route (Maguire et al. 1998, Hartley et al. 2003, Weniger et al. 2009, Weniger et al. 2010). Sometimes they should recall the landmark order or appearance along a known route (Jordan et al. 2004, Nemmi et al. 2013).

Mental navigation tasks require participants to imagine navigating in a very familiar environment. Moscovitch and his colleagues, for instance, asked subjects to mentally navigate in downtown Toronto where they had a vast experience navigating within 5-10 years prior to the experiment. In one of the egocentric tasks, participants had to establish whether the order of two landmarks along the route was correct if they were walking on the street named in the instruction in a particular direction. In the allocentric task, participants had to mentally imagine the configuration of three named landmarks in the city and report which of two landmarks was closer to the third one (Rosenbaum et al. 2004, Hirshhorn et al. 2012).

Another type of spatial task was based on the use of visual optic flow, which elicited in participants the feeling of passive transportation (Gramann et al. 2006, Gramann et al. 2010). First, subjects were passively transported through a tunnel consisted of straight and curved segments, and after that, they should determine the vector of direction relative to the starting point. Subjects responded spontaneously either relative to their heading in the last tunnel segment or relative to their heading at the beginning of the tunnel; besides, they mostly kept their responses consistent over the trials. The first type of response was interpreted as compatible with the use of an egocentric reference frame to keep track of their position. In the second type of response, according to authors, the subjects relied more on the allocentric representations, building the mental image of the whole virtual environment (a tunnel).

Despite the differences in these approaches, the studies revealed several common brain areas associated with egocentric and allocentric navigation. The fMRI and EEG analysis have shown that areas in the frontal, parietal, and occipital cortex were more active in tasks requiring the use of egocentric strategy (Jordan et al. 2004, Parslow et al. 2004, Rosenbaum et al. 2004, Gramann et al. 2006, Gramann et al. 2010, Weniger et al. 2010). In particular, an increase of BOLD signal was found in the precuneus and posterior parietal cortex (PPC), namely the inferior and superior parietal lobule (Parslow et al. 2004, Rosenbaum et al. 2004, Weniger et al. 2010), and also in the superior and middle frontal gyri (Rosenbaum et al. 2004, Weniger et al. 2010). A significant deactivation of the alpha band in subjects using egocentric strategy was also localized in the cuneus using scalp EEG recording (Gramann et al. 2010). Surprisingly, the parahippocampal cortex involved usually in allocentric representations demonstrated activation during navigation in the virtual maze without any landmarks in which participants presumably used egocentric strategy (Weniger et al. 2010). In the same virtual maze, patients with lesions to the right posterior parahippocampal gyrus also had difficulties finding the goal (Weniger and Irle 2006). Its activity may be connected with the analysis of the appearance of important navigation decision points (Janzen and van Turennout 2004).

The involvement of the PPC in egocentric navigation was repeatedly confirmed by studies of braindamaged patients. Aguirre and D'Esposito (1999) described patients with lesions to the superior parietal lobule who suffered from egocentric disorientation. They could not define the positions of objects and landmarks relative to themselves, and thus were often impaired in way-finding tasks, although their ability to recognize and identify objects and landmarks in the environment was preserved. Consistent with these findings, patients with the lesions to the PPC were also impaired in a series of egocentric mental tasks (Ciaramelli et al. 2010). For instance, they demonstrated impaired performance in a task requiring to imagine walking along the particular route in a very familiar environment (downtown Toronto) and judge the correctness of landmarks sequences. Besides, patients with the PPC lesions were impaired in navigation in the virtual maze with absent landmarks, which promoted the use of egocentric strategy (Weniger et al. 2009).

By contrast, allocentric-based navigation recruited mainly the hippocampus, parahippocampal gyrus and retrosplenial cortex across the experimental paradigms: in the virtual city or maze (Maguire et al. 1998, Jordan et al. 2004, Spiers and Maguire 2007, Iaria et al. 2007), virtual MWM (Parslow et al. 2004, Rodriguez 2010), and mental navigation (Rosenbaum et al. 2004, Hirshhorn et al. 2012). Clusters in the inferior parietal cortex, mostly pronounced in or near the precuneus, and in the retrosplenial cortex, 
were found to be selective for allocentric reference frame usage in an experiment with passive transportation in a tunnel in a scalp EEG study using analysis of eventrelated spectral perturbation and independent component clustering (Gramann et al. 2010). This parietal activation was interpreted as being connected with the visual imagery or maintaining concurrent egocentric and allocentric reference frame representations.

Lesions to the hippocampus, parahippocampal gyrus, and retrosplenial cortex also caused impairments in various aspects of allocentric navigation. For instance, lesions to the right retrosplenial region resulted in the heading disorientation (Aguirre and D'Esposito 1999) when patients could recognize salients landmarks but could not derive the directional information from them. This impairment was suggested to be associated with the role of the retrosplenial cortex in the translation between egocentric and allocentric representations (Byrne et al. 2007). After hippocampal lesions, patients suffered from topographical amnesia; i.e. they were unable to create memories of the new environment and navigate there (Aguirre and D'Esposito 1999, King et al. 2002, Hartley et al. 2007). Besides, patients with the damaged hippocampus (Astur et al. 2002, Feigenbaum and Morris 2004, Bartsch et al. 2010, Goodrich-Hunsaker et al. 2010) and parahippocampal cortex (Bohbot et al. 1998) were impaired in the allocentric condition in the MWM analogy.

Although the above-listed brain areas have shown a predominant activation during each type of navigation, both strategies often engage an overlapping network of brain regions. Both allocentric and egocentric tasks have elicited responses in the occipital, superior temporal, parietal, and frontal cortex (Parslow et al. 2004, Rosenbaum et al. 2004, Jordan et al. 2004, Gramann et al. 2010, Rodriguez 2010). Gramann et al. (2010) have found that only a small number of clusters of brain activity significantly differed between subjects relying on egocentric and allocentric reference frames. A recent meta-analysis of fMRI studies also revealed that allocentric and egocentric tasks share common brain areas (Boccia et al. 2014), including the middle occipital gyrus, cuneus, precuneus, fusiform and lingual gyri, precentral and middle frontal gyri with superior frontal lobe and insula. The explanation of this activation overlap may lie in the fact that during navigation, participants can use both strategies in parallel with one of them prevailing (Gramann 2013, Ekstrom et al. 2014). Furthermore, intersubject variability in the preferred strategy can also contribute to this overlap activation. For instance, in studies which allowed the utilization of both allocentric and egocentric strategy, participants differed in their preference to use one of them, called proclivity (Jordan et al. 2004, Gramann et al. 2006, Ohnishi et al. 2006, Igloi et al. 2009, Gramann et al. 2010).

A comparison between static and dynamic spatial studies indicates the common pattern of activation in the fronto-parietal network for the use of an egocentric reference frame (Table 1 and Fig. 1). The middle and superior frontal gyri showed activation in static twodimensional (Galati et al. 2000, Chen et al. 2012, Saj et al. 2014, Liu et al. 2017, Ruotolo et al. 2019), threedimensional tasks (Commiteri et al. 2004, Bastin et al. 2013) as well as during virtual and mental navigation (Rosenbaum et al. 2004, Weniger et al. 2010). The superior parietal lobule and precuneus were active during egocentric navigational tasks (Parslow et al. 2004, Rosenbaum et al. 2004, Weniger et al. 2010), in the egocentric frame transformations in static threedimensional scenes (Sulpizio et al. 2013) and also during egocentric judgments relative to the midsagittal body plane in two-dimensional images (Galati et al. 2000, Neggers et al. 2006, Chen et al. 2012, Saj et al. 2014, Liu et al. 2017, Ruotolo et al. 2019). Despite the similarity in neural activation, it is worth noting that all mentioned tasks do not entirely capture the nature of egocentric coding. In the real world, apart from visual information, the vestibular and proprioceptive stimuli contribute significantly to the forming of egocentric representations during selfmovements. The processing of this information is also involved in spatial updating, which occurs during natural movements and allows us to update the positions of surrounding objects relative to ourselves constantly. As it was shown, the precuneus plays a vital role in this process (Wolbers et al. 2008).

The neural correlates of allocentric coding in spatial navigational studies resemble those in the worldcentered allocentric condition in static studies using complex three-dimensional scenes (Table 1 and Fig. 1). In both cases, activation was found in the retrosplenial and parahippocampal cortex (Committeri et al. 2004, Bastin et al. 2013, Sulpizio et al. 2013, Maguire et al. 1998, Parslow et al. 2004, Rosenbaum et al. 2004, Jordan et al. 2004, Spiers and Maguire 2007, Rodriguez 2010). Both allocentric conditions, world-centered static and navigation, involved reorientation in a known environment after viewpoint changes associated with the retrosplenial activity. The parahippocampal activity is probably associated with spatial memory and scene analysis 
requirements of this condition. In comparison to the objectcentered condition in two- and three-dimensional studies, the response in the world-centered condition and navigation required processing of the whole scene and its components in the ventral visual stream. Besides, this world-centered condition in three-dimensional scenes involved spatial memory, in contrast to the object-centered condition where all elements of the task were directly presented. Particularly, participants should remember the configuration of landmarks in the whole environment to solve the task successfully. Similarly, tasks in spatial navigation studies require participants to use 'map' knowledge to navigate in the virtual environment flexibly.

Table 1. The summary of brain activation associated with the use of egocentric and allocentric reference frames across various experimental paradigms.

\begin{tabular}{|c|c|c|c|}
\hline \multirow{2}{*}{ Type of study } & \multirow{2}{*}{ Egocentric task } & \multicolumn{2}{|c|}{ Allocentric task } \\
\hline & & Object-centered & World-centered \\
\hline \multirow{10}{*}{ Static two-dimensional } & Parietal: & Occipital: & \\
\hline & SPL $\overline{(\text { predom } R H)}$ & $* \overline{\mathrm{IOG} \text { (both) }}$ & \\
\hline & IPL (predom RH) & Cun (both) & \\
\hline & IPS (both) & CalS (both) & \\
\hline & PCun (predom RH) & & \\
\hline & Frontal: & Temporal: & \\
\hline & $\mathrm{dPMC} \overline{\text { (predom } \mathrm{RH})}$ & $\overline{\mathrm{ITG}(\mathrm{LH})}$ & \\
\hline & SFG (predom RH) & * FuG (both) & \\
\hline & MFG (predom RH) & LG (both) & \\
\hline & IFG (predom RH) & & \\
\hline \multirow{10}{*}{ Static three-dimensional } & Occipital: & Occipital: & Parietal: \\
\hline & $\overline{\text { TPOJ (both) }}$ & $\begin{array}{c}\text { IOG (predom LH) } \\
\text { TPOJ (both) }\end{array}$ & PCun (predom LH) \\
\hline & Parietal: & Temporal: & Cingulate: \\
\hline & $\overline{\text { IPS (both) }}$ & ITG (predom RH) & $\overline{\mathrm{RSC} \text { (both) }}$ \\
\hline & PCun (both) & & \\
\hline & SMG (both) & & \\
\hline & Frontal: & & Temporal: \\
\hline & $\overline{\mathrm{SFG}(\mathrm{RH})}$ & & $\overline{\mathrm{FuG} \text { (both) }}$ \\
\hline & IFG (RH) & & LG (both) \\
\hline & & & PHG (RH) \\
\hline \multirow{9}{*}{ Spatial navigation } & Occipital: & & Cingulate: \\
\hline & $\overline{\text { Sun }(\mathrm{RH})}$ & & $\overline{\mathrm{RSC} \text { (both) }}$ \\
\hline & Parietal: & & Temporal: \\
\hline & PCun (both) & & PHG (both) \\
\hline & SPL (both) & & Hip (both) \\
\hline & IPL (both) & & \\
\hline & Frontal: & & \\
\hline & $\overline{\mathrm{SFG}(\mathrm{LH})}$ & & \\
\hline & MFG (RH) & & \\
\hline
\end{tabular}

The data were obtained mainly in fMRI studies. An asterisk $\left(^{*}\right)$ and a paragraph $\left(^{\S}\right)$ symbols indicate the activity of brain structures obtained in PET and EEG studies, respectively. In parentheses: RH and LH - activation found only in the right or left hemisphere, predom RH and predom LH - activation prevailed in the right or left hemisphere, both - bilateral activation. Abbreviations: CalS calcarine sulcus, Cun - cuneus, dPMC - dorsal premotor cortex, FuG - fusiform gyrus, Hip - hippocampus, IFG - inferior frontal gyrus, IOG - inferior occipital gyrus, IPL - inferior parietal lobule, IPS - intraparietal sulcus, ITG - inferior temporal gyrus, LG lingual gyrus, MFG - medial frontal gyrus, PCun - precuneus, PHG - parahippocampal gyrus, RSC - retrosplenial cortex, SFG superior frontal gyrus, SMG - supramarginal gyrus, SPL - superior parietal lobule, TPOJ - temporal-parietal-occipital junction. 
While navigation and world-centered static scene tasks shared the parahippocampal and retrosplenial cortex activity, the hippocampus seems to be involved only in navigation. Indeed, several studies have suggested that the hippocampus might not be necessary for 'simple' allocentric memory when there is no need for the processing and integration of multiple spatial locations either instantaneously in space or sequentially in time; instead, the parahippocampal cortex is involved (Bobhot et al. 1998, Bohbot and Corkin 2007, Zhang and Ekstrom 2013, for review see Burgess et al. 2002). Similarly, the hippocampus seems to be not involved in estimating position relative to a known landmark as in the worldcentered condition in the static tasks.

\section{A}

\section{Egocentric reference frame}

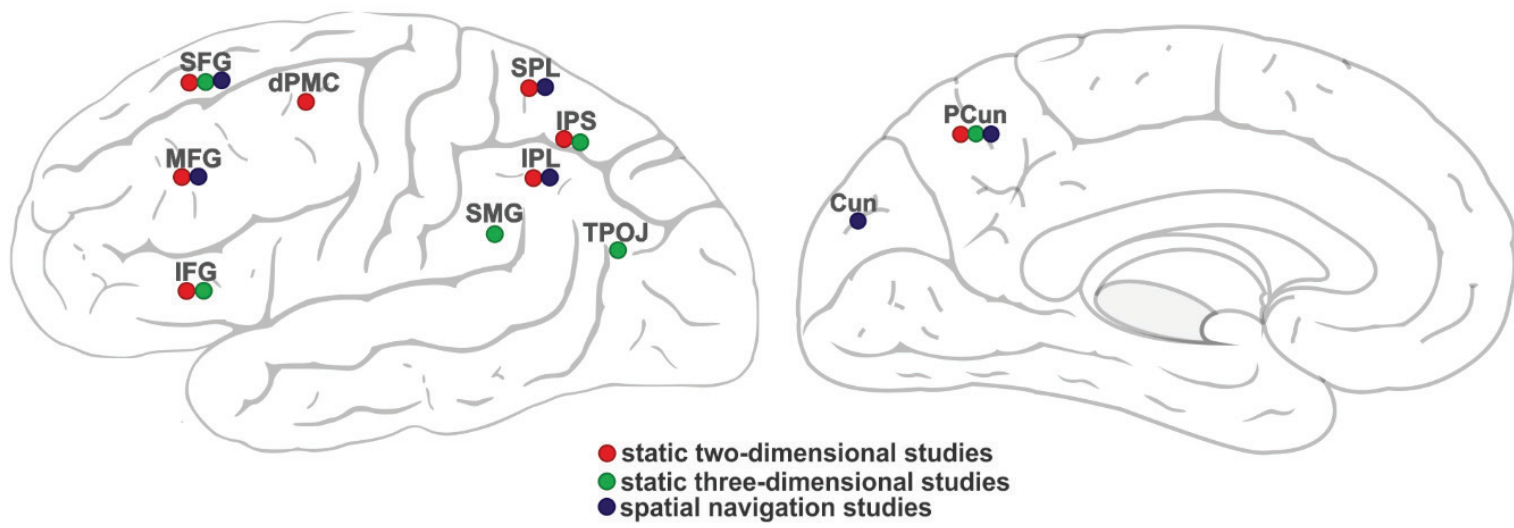

B

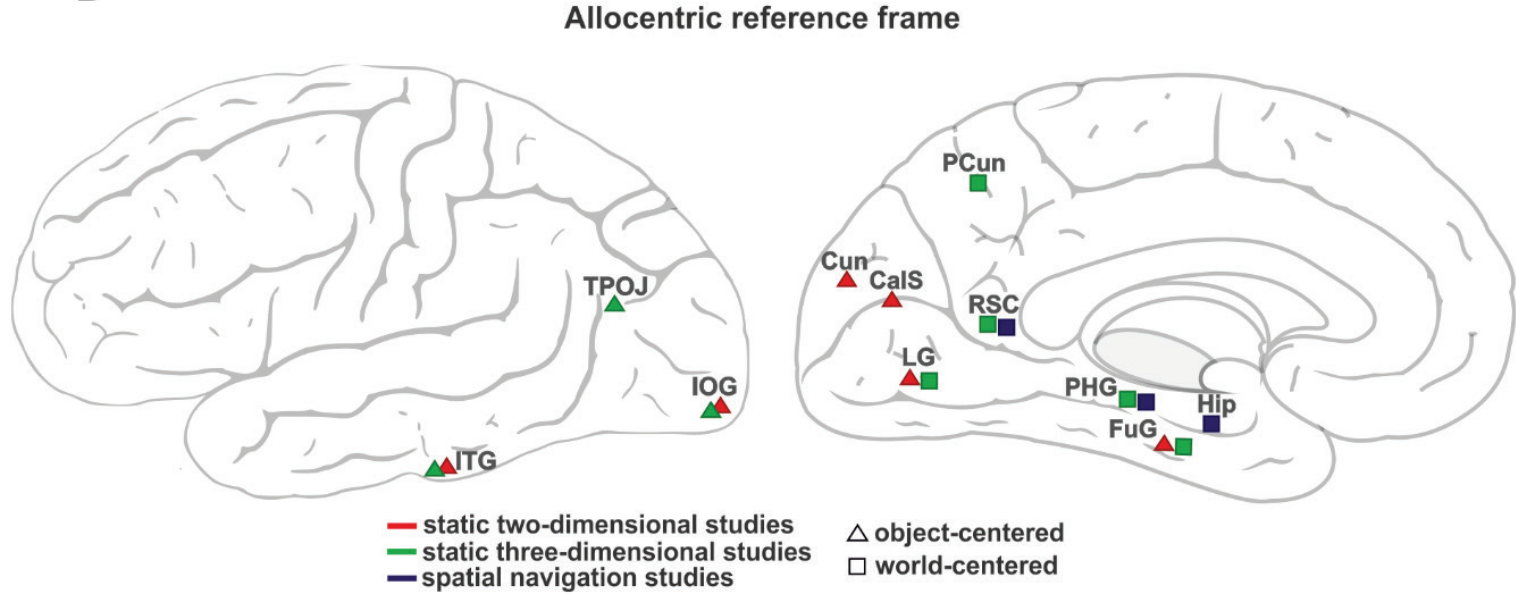

Fig. 1. A schematic overview of active areas in the brain associated with the use of spatial reference frames across three types of study: static two-dimensional (red color), static three-dimensional (green color) and spatial navigation (blue color). (A) the egocentric reference frame, involved brain areas are marked by circles, (B) the allocentric reference frame: the involved brain areas in objectcentered and world-centered reference frame are marked by triangles and squares, respectively. Abbreviations of the brain regions are the same as in Table 1. For simplicity, all symbols (circles, triangles and squares) represent the localization of brain regions schematically without reference to the lateralization and exact size and range of activation. The images of the brain were adapted from Wikimedia Commons (File: Gray726.svg and Gray727.svg), both in the public domain and authored by H. V. Carter.

\section{Comparison with other spatial navigation models}

The main findings from static and dynamic spatial studies partly correspond with the model of spatial memory and navigation proposed by Byrne et al. (2007, BBB model). According to this model, egocentric representations emerge originally in the PPC, while allocentric representations are stored in the medial temporal lobe (MTL), namely in the hippocampus and parahippocampal cortices. Brain activation found in static and spatial navigation studies during egocentric tasks seems to confirm the role of PPC in creating egocentric representations. On the other hand, a proposed role of 
MTL in the processing of allocentric information fits well only with the processing of world-centered allocentric representations, as it was shown in the static threedimensional and spatial navigation studies. In contrast, the object-centered representations seem to emerge in the ventral occipitotemporal region. The retrosplenial cortex, according to the BBB model, serves as a connecting hub between PPC and MTL and, at the same time, provides translation between egocentric and allocentric coordinates in both directions. The results of static three-dimensional and spatial navigation studies support this assumed function, as the retrosplenial activity was found only during the world-centered allocentric task, which involved spatial reorientation implying egocentricallocentric transformations.

\section{Conclusions}

Our review indicates several similarities and differences in neural correlates of allocentric and egocentric coding in humans across various experimental paradigms. First, a common activation pattern for the use of the egocentric reference frame is observed in both static tasks and spatial navigation. The fronto-parietal network showed activation during egocentric left/right judgments, visuomotor tasks performed in egocentric coordinates, egocentric transformations in threedimensional scenes, and also during the use of the egocentric strategy in virtual or mental navigation. Second, a neural pattern associated with the use of the allocentric object-centered reference frame in studies using a complex three-dimensional environment is comparable with that in two-dimensional tasks, both during perceptual spatial judgments and visuomotor tasks. In particular, the object-centered reference frame recruited parts of the inferior occipitotemporal region. Finally, allocentric coding involved in navigation is similar to the allocentric world-centered coding in static studies using a complex three-dimensional environment. In both cases, the response requires analyzing the spatial scene, reorienting in space, and defining spatial relationships relative to prominent features of the environment recalled from memory. The activation associated with these processes was found in the retrosplenial and parahippocampal cortex. Our review clarifies the involvement of the brain networks in the use of several types of spatial reference frames under various experimental paradigms. We document the distinct brain activity associated with two types of allocentric reference frames: the object-centered and world-centered. These findings suggest the need for further experiments aimed at differences in brain activity connected with the reference frames subtypes.

\section{Conflict of Interest}

There is no conflict of interest.

\section{Acknowledgements}

This work was supported by GACR grants 19-11753S and 16-07690S. Institutional support for IPHYS was provided by RVO: 67985823 .

\section{References}

AGUIRRE GK, D’ESPOSITO M: Topographical disorientation: A synthesis and taxonomy. Brain 122: 1613-1628, 1999. https://doi.org/10.1093/brain/122.9.1613

AGUIRRE GK, DETRE JA, ALSOP DC, D’ESPOSITO M: The parahippocampus subserves topographical learning in man. Cereb Cortex 6: 823-829, 1996. https://doi.org/10.1093/cercor/6.6.823

AMLEROVA J, LACZO J, VLCEK K, JAVURKOVA A, ANDEL R, MARUSIC P: Risk factors for spatial memory impairment in patients with temporal lobe epilepsy. Epilepsy Behav 26: 57-60, 2013. https://doi.org/10.1016/j.yebeh.2012.10.025

ASTUR RS, TAYLOR LB, MAMELAK AN, PHILPOTT L, SUTHERLAND RJ: Humans with hippocampus damage display severe spatial memory impairments in a virtual Morris water task. Behav Brain Res 132: 77-84, 2002. https://doi.org/10.1016/s0166-4328(01)00399-0

BARTSCH T, SCHÖNFELD R, MÜLLER FJ, ALFKE K, LEPLOW B, ALDENHOFF J, DEUSCHL G, KOCH JM: Focal lesions of human hippocampal CA1 neurons in transient global amnesia impair place memory. Science 328: 1412-1415, 2010. https://doi.org/10.1126/science.1188160 
BASTIN J, COMMITTERI G, KAHANE P, GALATI G, MINOTTI L, LACHAUX J-P, BERTHOZ A: Timing of posterior parahippocampal gyrus activity reveals multiple scene processing stages. Hum Brain Mapp 34: 1357-1370, 2013. https://doi.org/10.1002/hbm.21515

BEURZE SM, TONI I, PISELLA L, MEDENDORP WP: Reference frames for reach planning in human parietofrontal cortex. J Neurophysiol 104: 1736-1745, 2010. https://doi.org/10.1152/jn.01044.2009

BOCCIA M, NEMMI F, GUARIGLIA C: Neuropsychology of environmental navigation in humans: Review and metaanalysis of fMRI studies in healthy participants. Neuropsychol Rev 24: 236251, 2014. https://doi.org/10.1007/s11065-014-9247-8

BOHBOT VD, CORKIN S: Posterior parahippocampal place learning in H.M. Hippocampus 17: 863-872, 2007. https://doi.org/10.1002/hipo.20313

BOHBOT VD, KALINA M, STEPANKOVA K, SPACKOVA N, PETRIDES M, NADEL L: Spatial memory deficits in patients with lesions to the right hippocampus and to the right parahippocampal cortex. Neuropsychologia 36: 1217-1238, 1998. https://doi.org/10.1016/s0028-3932(97)00161-9

BURGESS N, MAGUIRE EA, O'KEEFE J: The human hippocampus and spatial and episodic memory. Neuron 35: 625-641, 2002. https://doi.org/10.1016/S0896-6273(02)00830-9

BYRNE P, BECKER S, BURGESS N: Remembering the past and imagining the future: A neural model of spatial memory and imagery. Psychol Rev 114: 340-375, 2007. https://doi.org/10.1037/0033-295X.114.2.340

CHECHLACZ M, ROTSHTEIN P, HUMPHREYS GW: Neuroanatomical dissections of unilateral visual neglect symptoms: ALE meta-analysis of lesion-symptom mapping. Front Hum Neurosci 6: 230, 2012. https://doi.org/10.3389/fnhum.2012.00230

CHEN Q, WEIDNER R, WEISS PH, MARSHALL JC, FINK GR: Neural interaction between spatial domain and spatial reference frame in parietal-occipital junction. J Cogn Neurosci 24: 2223-2236, 2012. https://doi.org/10.1162/jocn_a 00260

CHEN Y, MONACO S, BYRNE P, YAN X, HENRIQUES DYP, CRAWFORD JD: Allocentric versus egocentric representation of remembered reach targets in human cortex. J Neurosci 34: 12515, 2014. https://doi.org/10.1523/JNEUROSCI.1445-14.2014

CHEN Y, MONACO S, CRAWFORD JD: Neural substrates for allocentric-to-egocentric conversion of remembered reach targets in humans. Eur J Neurosci 47: 901-917, 2018. https://doi.org/10.1111/ejn.13885

CIARAMELLI E, ROSENBAUM RS, SOLCZ S, LEVINE B, MOSCOVITCH M: Mental space travel: Damage to posterior parietal cortex prevents egocentric navigation and reexperiencing of remote spatial memories. J Exp Psychol Learn Mem Cogn 36: 619-634, 2010. https://doi.org/10.1037/a0019181

COMMITTERI G, GALATI G, PARADIS A-L, PIZZAMIGLIO L, BERTHOZ A, LEBIHAN D: Reference frames for spatial cognition: Different brain areas are involved in viewer-, object-, and landmark-centered judgments about object location. J Cogn Neurosci 16: 1517-1535, 2004. https://doi.org/10.1162/0898929042568550

DEMEYERE N, GILLEBERT CR: Ego- and allocentric visuospatial neglect: Dissociations, prevalence, and laterality in acute stroke. Neuropsychology 33: 490-498, 2019. https://doi.org/10.1037/neu0000527

DHINDSA K, DROBININ V, KING J, HALL GB, BURGESS N, BECKER S: Examining the role of the temporoparietal network in memory, imagery, and viewpoint transformations. Front Hum Neurosci 8: 1-12, 2014. https://doi.org/10.3389/fnhum.2014.00709

EASTON RD, SHOLL MJ: Object-array structure, frames of reference, and retrieval of spatial knowledge. J Exp Psychol Learn Mem Cogn 21: 483-500, 1995. https://doi.org/10.1037//0278-7393.21.2.483

EKSTROM AD, ARNOLD AEGF, IARIA G: A critical review of the allocentric spatial representation and its neural underpinnings: toward a network-based perspective. Front Hum Neurosci 8: 1-15, 2014. https://doi.org/10.3389/fnhum.2014.00803

EKSTROM AD, HUFFMAN DJ, STARRETT M: Where are you going? The neurobiology of navigation: Interacting networks of brain regions underlie human spatial navigation: a review and novel synthesis of the literature. J Neurophysiol 118: 3328-3344, 2017. https://doi.org/10.1152/jn.00531.2017

EPSTEIN R, KANWISHER N: A cortical representation of the local visual environment. Nature 392: 598-601, 1998. https://doi.org/10.1038/33402 
FEIGENBAUM JD, MORRIS RG: Allocentric versus egocentric spatial memory after unilateral temporal lobectomy in humans. Neuropsychology 18: 462-472, 2004. https://doi.org/10.1037/0894-4105.18.3.462

FLETCHER PC, SHALLICE T, FRITH CD, FRACKOWIAK RSJ, DOLAN RJ: Brain activity during memory retrieval: The influence of imagery and semantic cueing. Brain 119: 1587-1596, 1996. https://doi.org/10.1093/brain/119.5.1587

GALATI G, LOBEL E, VALLAR G, BERTHOZ A, PIZZAMIGLIO L, LE BIHAN D: The neural basis of egocentric and allocentric coding of space in humans: a functional magnetic resonance study. Exp Brain Res 133: 156-164, 2000. https://doi.org/10.1007/s002210000375

GHAEM O, MELLET E, CRIVELLO F, TZOURIO N, MAZOYER B, BERTHOZ A, DENIS M: Mental navigation along memorized routes activates the hippocampus, precuneus, and insula. Neuroreport 8: 739-744, 1997. https://doi.org/10.1097/00001756-199702100-00032

GOODALE MA, MILNER AD: Separate visual pathways for perception and action. Trends Neurosci 15: 20-25, 1992. https://doi.org/10.1016/0166-2236(92)90344-8

GOODRICH-HUNSAKER NJ, LIVINGSTONE SA, SKELTON RW, HOPKINS RO: Spatial deficits in a virtual water maze in amnesic participants with hippocampal damage. Hippocampus 20: 481-491, 2010. https://doi.org/10.1002/hipo.20651

GRAMANN K: Embodiment of spatial reference frames and individual differences in reference frame proclivity. Spat Cogn Comput 13: 1-25, 2013. https://doi.org/10.1080/13875868.2011.589038

GRAMANN K, MÜLLER HJ, SCHÖNEBECK B, DEBUS G: The neural basis of ego- and allocentric reference frames in spatial navigation: Evidence from spatio-temporal coupled current density reconstruction. Brain Res 1118: 116-129, 2006. https://doi.org/10.1016/j.brainres.2006.08.005

GRAMANN K, ONTON J, RICCOBON D, MUELLER HJ, BARDINS S, MAKEIG S: Human brain dynamics accompanying use of egocentric and allocentric reference frames during navigation. J Cogn Neurosci 22: 2836-2849, 2010. https://doi.org/10.1162/jocn.2009.21369

GRIMSEN C, HILDEBRANDT H, FAHLE M: Dissociation of egocentric and allocentric coding of space in visual search after right middle cerebral artery stroke. Neuropsychologia 46: 902-914, 2008. https://doi.org/10.1016/j.neuropsychologia.2007.11.028

HARTLEY T, BIRD CM, CHAN D, CIPOLOTTI L, HUSAIN M, VARGHA-KHADEM F, BURGESS N: The hippocampus is required for short-term topographical memory in humans. Hippocampus 17: 34-48, 2007. https://doi.org/10.1002/hipo.20240

HARTLEY T, MAGUIRE EA, SPIERS HJ, BURGESS N: The well-worn route and the path less traveled: Distinct neural bases of route following and wayfinding in humans. Neuron 37: 877-888, 2003. https://doi.org/10.1016/S0896-6273(03)00095-3

HIRSHHORN M, GRADY C, ROSENBAUM RS, WINOCUR G, MOSCOVITCH M: The hippocampus is involved in mental navigation for a recently learned, but not a highly familiar environment: A longitudinal fMRI study. Hippocampus 22: 842-852, 2012. https://doi.org/10.1002/hipo.20944

HONDA M, WISE SP, WEEKS RA, DEIBER MP, HALLETT M: Cortical areas with enhanced activation during object-centred spatial information processing. A PET study. Brain 121: 2145-2158, 1998. https://doi.org/10.1093/brain/121.11.2145

IARIA G, CHEN J-K, GUARIGLIA C, PTITO A, PETRIDES M: Retrosplenial and hippocampal brain regions in human navigation: complementary functional contributions to the formation and use of cognitive maps. Eur J Neurosci 25: 890-899, 2007. https://doi.org/10.1111/j.1460-9568.2007.05371.x

IGLOI K, ZAOUI M, BERTHOZ A, RONDI-REIG L: Sequential egocentric strategy is acquired as early as allocentric strategy: Parallel acquisition of these two navigation strategies. Hippocampus 19: 1199-1211, 2009. https://doi.org/10.1002/hipo.20595

ISHIAI S, FURUKAWA T, TSUKAGOSHI H: Visuospatial processes of line bisection and the mechanisms underlying unilateral spatial neglect. Brain 112: 1485-1502, 1989. https://doi.org/10.1093/brain/112.6.1485

JANZEN G, VAN TURENNOUT M: Selective neural representation of objects relevant for navigation. Nat Neurosci 7 : 673-677, 2004. https://doi.org/10.1038/nn1257 
JORDAN K, SCHADOW J, WUESTENBERG T, HEINZE H-J, JÄNCKE L: Different cortical activations for subjects using allocentric or egocentric strategies in a virtual navigation task. Neuroreport 15: 135-140, 2004. https://doi.org/10.1097/00001756-200401190-00026

JULIAN JB, KEINATH AT, MARCHETTE SA, EPSTEIN RA: The neurocognitive basis of spatial reorientation. Curr Biol 28: R1059-R1073, 2018. https://doi.org/10.1016/j.cub.2018.04.057

KALOVÁ E, VLCEK K, JAROLÍMOVÁ E, BURES J: Allothetic orientation and sequential ordering of places is impaired in early stages of Alzheimer's disease: corresponding results in real space tests and computer tests. Behav Brain Res 159: 175-186, 2005. https://doi.org/10.1016/j.bbr.2004.10.016

KENZIE JM, GIRGULIS KA, SEMRAU JA, FINDLATER SE, DESAI JA, DUKELOW SP: Lesion sites associated with allocentric and egocentric visuospatial neglect in acute stroke. Brain Connect 5: 413-422, 2015. https://doi.org/10.1089/brain.2014.0316

KING JA, BURGESS N, HARTLEY T, VARGHA-KHADEM F, O'KEEFE J: Human hippocampus and viewpoint dependence in spatial memory. Hippocampus 12: 811-820, 2002. https://doi.org/10.1002/hipo.10070

KLATZKY RL: Allocentric and egocentric spatial representations: Definitions, distinctions, and interconnections. In: Lecture Notes in Computer Science 1404; Spatial Cognition: An Interdisciplinary Approach to Representing and Processing Spatial Knowledge. FREKSA C, HABEL C, WENDER KF (eds), Springer, Berlin, Heidelberg, 1998, pp 1-17. https://doi.org/10.1007/3-540-69342-4_1

LIU N, LI H, SU W, CHEN Q: Common and specific neural correlates underlying the spatial congruency effect induced by the egocentric and allocentric reference frame: Spatial conflict between spatial reference frames. Hum Brain Mapp 38: 2112-2127, 2017. https://doi.org/10.1002/hbm.23508

MAGUIRE EA, BURGESS N, DONNETT JG, FRACKOWIAK RS, FRITH CD, O'KEEFE J: Knowing where and getting there: a human navigation network. Science 280: 921-924, 1998. https://doi.org/10.1126/science.280.5365.921

MAKIN TR, HOLMES NP, ZOHARY E: Is that near my hand? Multisensory representation of peripersonal space in human intraparietal sulcus. J Neurosci 27: 731-740, 2007. https://doi.org/10.1523/JNEUROSCI.3653-06.2007

MALOUIN F, RICHARDS CL, JACKSON PL, DUMAS F, DOYON J: Brain activations during motor imagery of locomotor-related tasks: A PET study. Hum Brain Mapp 19: 47-62, 2003. https://doi.org/10.1002/hbm.10103

MEDENDORP WP, GOLTZ HC, VILIS T, CRAWFORD JD: Gaze-centered updating of visual space in human parietal cortex. J Neurosci 23: 6209-6214, 2003. https://doi.org/10.1523/JNEUROSCI.23-15-06209.2003

NEGGERS SFW, VAN DER LUBBE RHJ, RAMSEY NF, POSTMA A: Interactions between ego- and allocentric neuronal representations of space. Neuroimage 31: 320-331, 2006. https://doi.org/10.1016/j.neuroimage.2005.12.028

NEMMI F, PIRAS F, PÉRAN P, INCOCCIA C, SABATINI U, GUARIGLIA C: Landmark sequencing and route knowledge: An fMRI study. Cortex 49: 507-519, 2013. https://doi.org/10.1016/j.cortex.2011.11.016

OHNISHI T, MATSUDA H, HIRAKATA M, UGAWA Y: Navigation ability dependent neural activation in the human brain: An fMRI study. Neurosci Res 55: 361-369, 2006. https://doi.org/10.1016/j.neures.2006.04.009

O'KEEFE J, NADEL L: The Hippocampus as a Cognitive Map. Oxford: Clarendon Press, 1978, 569 p.

PARSLOW DM, ROSE D, BROOKS B, FLEMINGER S, GRAY JA, GIAMPIETRO V, BRAMMER MJ, WILLIAMS S, GASSTON D, ANDREW C, VYTHELINGUM GN, LOANNOU G, SIMMONS A, MORRIS RG: Allocentric spatial memory activation of the hippocampal formation measured with fMRI. Neuropsychology 18: 450-461, 2004. https://doi.org/10.1037/0894-4105.18.3.450

PATCHAY S, HAGGARD P, CASTIELLO U: An object-centred reference frame for control of grasping: effects of grasping a distractor object on visuomotor control. Exp Brain Res 170: 532-542, 2006. https://doi.org/10.1007/s00221-005-0240-6

RODRIGUEZ PF: Human navigation that requires calculating heading vectors recruits parietal cortex in a virtual and visually sparse water maze task in fMRI. Behav Neurosci 124: 532-540, 2010. https://doi.org/10.1037/a0020231

ROSENBAUM RS, ZIEGLER M, WINOCUR G, GRADY CL, MOSCOVITCH M: "I have often walked down this street before": fMRI Studies on the hippocampus and other structures during mental navigation of an old environment. Hippocampus 14: 826-835, 2004. https://doi.org/10.1002/hipo.10218 
RUOTOLO F, RUGGIERO G, RAEMAEKERS M, IACHINI T, VAN DER HAM IJM, FRACASSO A, POSTMA A: Neural correlates of egocentric and allocentric frames of reference combined with metric and non-metric spatial relations. Neuroscience 409: 235-252, 2019. https://doi.org/10.1016/j.neuroscience.2019.04.021

SAJ A, COJAN Y, MUSEL B, HONORÉ J, BOREL L, VUILLEUMIER P: Functional neuro-anatomy of egocentric versus allocentric space representation. Neurophysiol Clin 44: 33-40, 2014. https://doi.org/10.1016/j.neucli.2013.10.135

SCHENKENBERG T, BRADFORD DC, AJAX ET: Line bisection and unilateral visual neglect in patients with neurologic impairment. Neurology 30: 509-517, 1980. https://doi.org/10.1212/wnl.30.5.509

SERENO MI, HUANG R-S: A human parietal face area contains aligned head-centered visual and tactile maps. Nat Neurosci 9: 1337-1343, 2006. https://doi.org/10.1038/nn1777

SPIERS HJ, MAGUIRE EA: A navigational guidance system in the human brain. Hippocampus 17: 618-626, 2007. https://doi.org/10.1002/hipo.20298

SULPIZIO V, COMMITTERI G, LAMBREY S, BERTHOZ A, GALATI G: Selective role of lingual/parahippocampal gyrus and retrosplenial complex in spatial memory across viewpoint changes relative to the environmental reference frame. Behav Brain Res 242: 62-75, 2013. https://doi.org/10.1016/j.bbr.2012.12.031

VALLAR G: Spatial Neglect, Balint-Homes' and Gerstmann's syndrome, and other spatial disorders. CNS Spectr 12: 527-536, 2007. https://doi.org/10.1017/S1092852900021271

WENIGER G, IRLE E: Posterior parahippocampal gyrus lesions in the human impair egocentric learning in a virtual environment. Eur J Neurosci 24: 2406-2414, 2006. https://doi.org/10.1111/j.1460-9568.2006.05108.x

WENIGER G, RUHLEDER M, WOLF S, LANGE C, IRLE E: Egocentric memory impaired and allocentric memory intact as assessed by virtual reality in subjects with unilateral parietal cortex lesions. Neuropsychologia 47: 59-69, 2009. https://doi.org/10.1016/j.neuropsychologia.2008.08.018

WENIGER G, SIEMERKUS J, SCHMIDT-SAMOA C, MEHLITZ M, BAUDEWIG J, DECHENT P, IRLE E: The human parahippocampal cortex subserves egocentric spatial learning during navigation in a virtual maze. Neurobiol Learn Mem 93: 46-55, 2010. https://doi.org/10.1016/j.nlm.2009.08.003

WOLBERS T, HEGARTY M, BÜCHEL C, LOOMIS JM: Spatial updating: how the brain keeps track of changing object locations during observer motion. Nat Neurosci 11: 1223-1230, 2008. https://doi.org/10.1038/nn.2189

WOLBERS T, WIENER JM: Challenges for identifying the neural mechanisms that support spatial navigation: the impact of spatial scale. Front Hum Neurosci 8: 1-12, 2014. https://doi.org/10.3389/fnhum.2014.00571

ZHANG H, EKSTROM A: Human neural systems underlying rigid and flexible forms of allocentric spatial representation. Hum Brain Mapp 34: 1070-1087, 2013. https://doi.org/10.1002/hbm.21494 